\title{
Novel conductor design to increase the thermal rating of overhead lines
}

\author{
J.C. del-Pino-López, D. Garrido-García, P. Cruz-Romero and A. Gómez-Expósito \\ Department of Electrical Engineering \\ Escuela Técnica Superior de Ingeniería, Universidad de Sevilla \\ Camino de los Descubrimientos s/n, 41092 Sevilla (Spain) \\ Phone/Fax number:+0034 954552847, e-mail: vaisat@us.es
}

\begin{abstract}
In this paper the performance of existing and novel designs of overhead bare conductors with regard to their capacity to evacuate the heat generated by ohmic losses is compared. The effect of both the diameter of strands and the external shape of the conductor on the convective heat transfer capacity are evaluated with a finite element method commercial software for an ACSR conductor with different diameter strands, wind velocities and conductor current, resulting in a design that improves the customary design by around a $7 \%$ of thermal rating.
\end{abstract}

\section{Key words}

Overhead conductor, ampacity, temperature, forced convection, radiation.

\section{Introduction}

The thermal rating of overhead line bare conductors is limited by either the minimum clearance above ground, as required by specific technical regulation, or the creep process, which accelerates with temperature. The vast majority of HV overhead lines and the shortest EHV lines have their transmission capacity thermally limited, so different techniques have been developed to uprate them, like dynamic line rating (DLR) [1], re-conductoring with low-sag conductors [2], and re-tensioning.

One of the ambient parameters that critically affect the conductor temperature is the wind velocity through the forced convection process [3]. This circumstance is opening the possibility to develop DLR tailored to the evacuation lines of wind farms, where an increase of power is correlated with a higher wind velocity [4].

The forced convection is not only affected by the wind speed, but also by the surface and shape of the conductor, so that a favourable conductor design could increase the heat evacuated by forced convection.

In this paper, we continue the research started in [5] with the aim of designing new overhead conductors that have a better convective heat transfer performance. The problem involved includes three coupled physics phenomena: electromagnetic, thermal, and fluid dynamics. To solve this problem, a good choice is to resort to multi-physics software like COMSOL [6], in order to compute the temperature inside the conductor with enough accuracy.

The use of conductor shapes other than circular has been proposed previously, not for the purpose of increasing the ampacity, but to reduce the wind load on the conductor [7].

The paper is structured as follows. In section 2 the multiphysics problem is formulated. Section 3 analyzes the effect of the outermost layer design for a particular conductor. In section 4 the effect of the conductor shape is studied. Finally, in section 5 the conclusions are presented.

\section{Problem definition}

In the following, the main hypotheses considered, as well as the mathematical models to be solved, are described [8].

\section{A. Electromagnetic problem}

To compute the heat dissipated in the power conductor for a given current, the electromagnetic problem needs to be solved. Its value is the heat input for the thermal problem. To this aim the following assumptions are considered:

1) The power conductor is straight and infinitely long.

2) The phase current is sinusoidal with $50 \mathrm{~Hz}$ of frequency.

3) All materials have constant electrical properties, with the exception of conductive materials, whose electrical conductivity $\sigma(\theta)$ depends on temperature:

$$
\sigma(\theta)=\frac{\sigma_{0}}{1+\alpha_{c}(\theta-20)},
$$

where $\theta$ is the unknown temperature and $\sigma_{0}$ and $\alpha$ are the conductivity and the temperature coefficient of the material at $20^{\circ} \mathrm{C}$, respectively.

4) Ferromagnetic materials are supposed to have constant permeability $(\mu)$.

5) Magnetics losses in ferromagnetic materials are neglected. 
In this situation, the equation to be solved can be described as

$$
\nabla \times\left(\frac{1}{\mu} \nabla \times \vec{A}\right)+j \omega \sigma \vec{A}=\vec{J}_{e},
$$

being $\vec{A}$ the magnetic vector potential, $\omega$ the angular frequency and $\vec{J}_{e}$ the external current density.

Once the problem is solved, the power losses generated in the conductor $\left(Q_{l}\right)$ can be calculated from the total current density $\vec{J}$ as

$$
Q_{l}=\int \frac{\vec{J} \cdot \vec{J}^{*}}{\sigma} d S .
$$

\section{B. Thermal problem}

The thermal problem is solved by considering the following assumptions:

1) Since the power cable is straight and infinitely long, the heat transfer problem can be formulated in $2 \mathrm{D}$ on the $x$ - $y$ plane.

2) All materials have constant thermal properties.

3) The air temperature is known $\left(\theta_{a m b}\right)$.

4) Heat radiation $\left(Q_{\mathrm{rad}}\right)$ is present between the outer cable surface and the surrounding environment, with known surface emissivity $(\varepsilon)$ of the materials.

5) Solar heating is present $\left(Q_{s}\right)$, with known solar radiation $\left(I_{s}\right)$ and surface absorptivity $\left(\alpha_{s}\right)$ of the materials.

6) Heat dissipation by forced convection is also considered $\left(\mathrm{Q}_{\text {conv }}\right)$.

7) Wind velocity is known and perpendicular to the power conductor, so that the system remains in 2D.

In consequence, the temperature of the power conductor can be derived from the associated steady-state heat balance equation, expressed as

$$
Q_{l}+Q_{s}=Q_{c o n v}+Q_{r a d}
$$

where steady-state heat conduction inside the conductor is given by

$$
\nabla \cdot(k \nabla \theta)+Q_{l}=0
$$

the radiation heat loss is derived from

$$
Q_{\text {rad }}=\varepsilon \cdot \sigma \cdot\left(\theta^{4}-\theta_{a m b}^{4}\right)
$$

the heat input caused by solar radiation in a circular conductor is obtained from

$$
Q_{S}=\alpha_{s} \cdot D \cdot I_{S} \cdot \sin \varphi
$$

and the heat dissipated by forced convection is obtained from

$$
\rho C_{p} u \cdot \nabla \theta=\nabla \cdot(k \nabla \theta)
$$

being $\rho$ the air density, $C_{p}$ the heat capacity of air, $u$ the air velocity vector, $\theta$ the unknown temperature, $D$ the conductor diameter, $\varphi$ the elevation angle of the Sun, $\sigma$ the Stefan-Boltzmann constant, and $k$ the thermal conductivity of each material.

Since there are temperature-sensitive properties, such as the electrical conductivity of the conductor, the coupled electromagnetic-thermal problem is iteratively solved. Hence, these parameters are continuously corrected for the newly calculated temperature.

\section{Fluid dynamics problem}

For the computation of the heat transferred by forced convection, the air velocity field $u$ around the conductor is required. This involves the computation of the continuity and momentum equations (Navier-Stokes equations) that govern the fluid flow, in the form of

$$
\begin{gathered}
\nabla \cdot(\rho u)=0, \\
\rho u \cdot \nabla u=-\nabla p+\nabla \cdot\left(\mu\left(\nabla u+(\nabla u)^{T}\right)\right),
\end{gathered}
$$

where $p$ and $\mu$ are the air pressure and viscosity, respectively. Since air properties are temperature dependent also, the fully coupled problem must be solved employing iterative techniques. As wind velocity ranges from $0.5 \mathrm{~m} / \mathrm{s}$ to $10 \mathrm{~m} / \mathrm{s}$, a $k-\varepsilon$ turbulence model was considered.

\section{Effect of the outermost layer design}

The case of the ACSR round conductor with $400 \mathrm{~mm}^{2} \mathrm{Al}$ and $25 \mathrm{~mm}^{2}$ St is analyzed next, where the properties of the materials are included in Table I. Regarding the boundary conditions, the temperature of the air is taken as $\theta_{a m b}=20^{\circ} \mathrm{C}$, for a wind velocity $\left(V_{i n}\right)$ ranging from 0.5 $\mathrm{m} / \mathrm{s}$ to $10 \mathrm{~m} / \mathrm{s}$ (wind blowing in the horizontal direction). The solar radiation is taken as $I_{s}=1000 \mathrm{~W} / \mathrm{m}^{2}$, with the sun placed directly over the conductor $\left(\varphi=90^{\circ}\right)$. Finally, the current through the conductor $\left(I_{0}\right)$ will vary from 600 A to $2500 \mathrm{~A}$.

Table I. - Material properties.

\begin{tabular}{|c|c|}
\hline Material & Property \\
\hline & $\sigma_{0}=3.77 \cdot 10^{7} \mathrm{~S} / \mathrm{m}$ \\
Aluminium & $\alpha=0.00403^{\circ} \mathrm{C}^{-1}$ \\
(Al) & $\mu_{r}=1$ \\
& $k=240 \mathrm{~W} / \mathrm{mK}$ \\
$\varepsilon=\alpha_{s}=0.8$ \\
\hline & $\sigma_{0}=4 \cdot 10^{6} \mathrm{~S} / \mathrm{m}$ \\
& $\alpha_{c}=0.005^{\circ} \mathrm{C}^{-1}$ \\
& $\mu_{r}=300$ \\
Steel (St) & $k=56 \mathrm{~W} / \mathrm{mK}$ \\
& Properties from \\
& COMSOL library \\
\hline
\end{tabular}

For the sake of simplicity, the geometry of the power conductor is as shown in Fig. 1 [9]. Since Al and St strands are all in contact, the temperature inside the conductor is quite uniform. As a consequence, strands are taken into account to model the surface of the conductor 
only. Also, the steel reinforcement is assumed to have circular cross section.

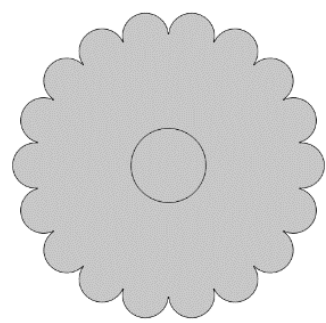

Fig 1 . Geometry of the conductor.

In this situation, for a given current (800 A) and wind velocity $(1 \mathrm{~m} / \mathrm{s})$, the temperature and velocity fields around the conductor are shown in Fig. 2. The Sun is supposed to be at the zenith $\left(\varphi=90^{\circ}\right)$.

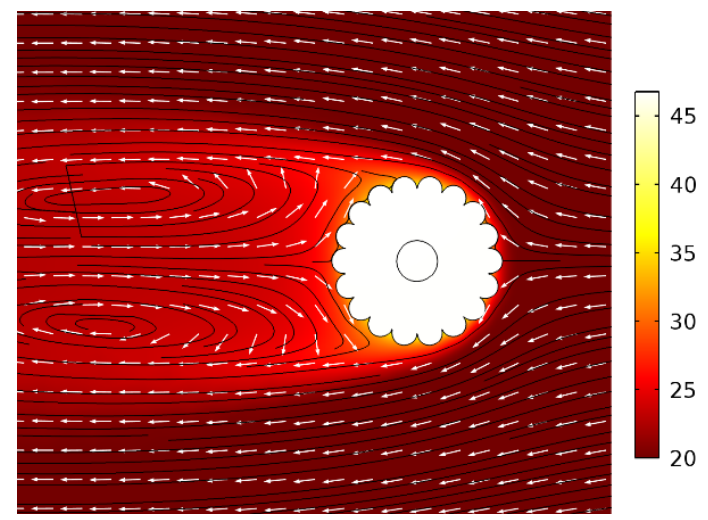

Fig. 2. Temperature (in ${ }^{\circ} \mathrm{C}$ ) and velocity field around the conductor (current of $800 \mathrm{~A}$ and wind velocity of $1 \mathrm{~m} / \mathrm{s}$ ).

Regarding the strands, their dimension may have a strong influence on the heat transfer mechanisms present in this model. In particular, depending on their diameter, the surface exposed to radiation, convection and solar heating is different, and so the heat evacuated from the conductor. In this sense, Fig. 3 shows the evolution of the maximum temperature obtained in the conductor as a function of strand diameter and wind velocity.

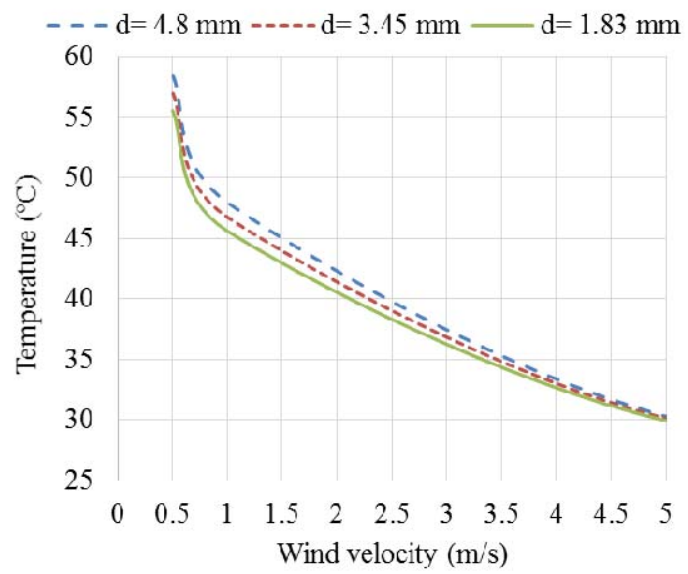

Fig. 3. Influence of $\mathrm{Al}$ strands diameter on the maximum temperature of the conductor for different wind velocities (current of $800 \mathrm{~A}$ ).

As can be observed, for a given wind velocity, the temperature of the conductor may be more than $2.5^{\circ} \mathrm{C}$ higher when using strands of $4.8 \mathrm{~mm}$ instead of $1.83 \mathrm{~mm}$, although this behaviour is more important for wind velocities in the range of $0.5-2 \mathrm{~m} / \mathrm{s}$. This is more detailed in Fig. 4 for a wind velocity of $1 \mathrm{~m} / \mathrm{s}$. The figure shows that reducing the strand diameter leads to lower temperatures, even lower than that obtained when considering a circular non-stranded conductor (smooth) (Fig. 5). The lower the strand diameter, the higher the perimeter of the conductor, hence the higher surface exposed to forced convection and heat radiation. An additional parameter that could improve even further the heat evacuation is the conductor shape, being analyzed next.

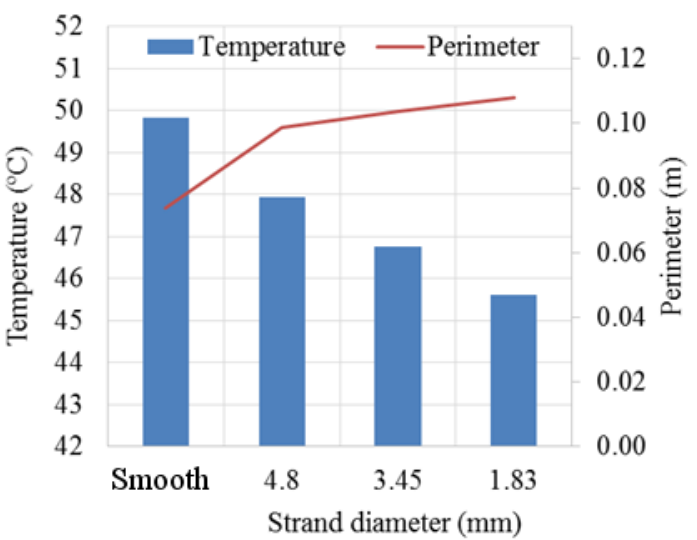

Fig. 4. Maximum temperature for different strand diameters and the smooth case (current of $800 \mathrm{~A}$ and wind velocity of $1 \mathrm{~m} / \mathrm{s}$ ).

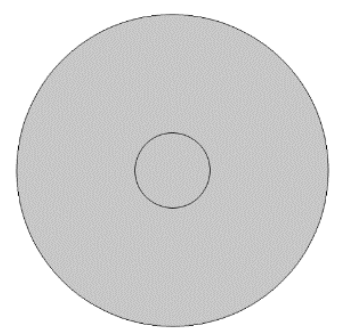

Fig. 5. Perfect circular conductor (smooth).

\section{Effect of conductor shape}

In [5], two possibilities were presented in relation with the position of the elliptical conductor, horizontal and vertical (Fig. 6).

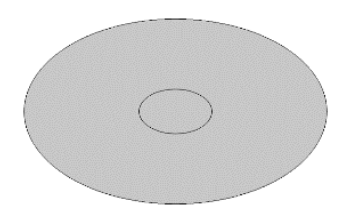

(a)

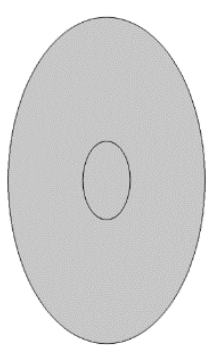

(b)
Fig. 6. Simplified geometry of horizontal (a) and vertical (b) elliptical conductor.

In a similar way to the circular conductor, the geometry of the elliptical conductor may be simplified, only considering the strands to model the surface of the conductor. This way, Fig. 7 shows the temperature and velocity fields around elliptical conductors (major axis- 
to-minor axis ratio of 1.4) of the same $\mathrm{Al}$ and St sections and the same current and wind velocity than in Fig. 2.

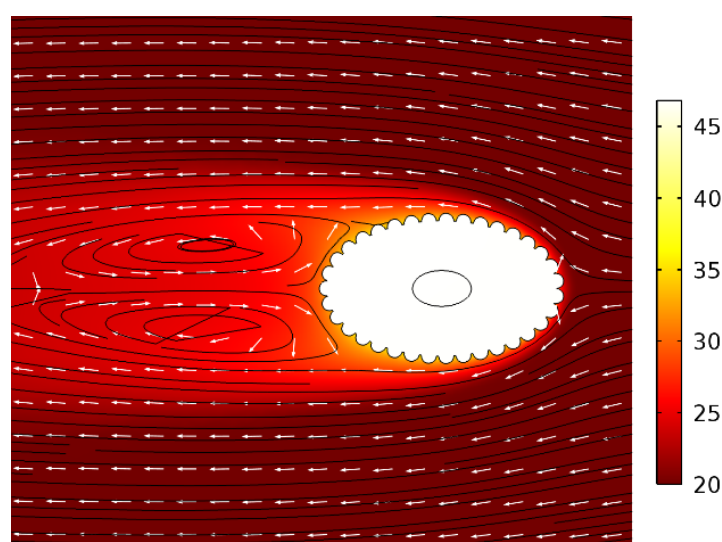

(a)

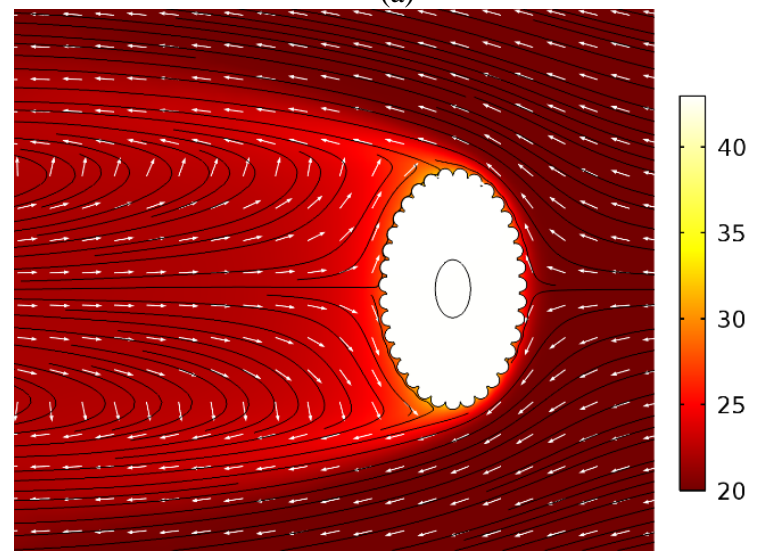

(b)

Fig. 7. Temperature (in ${ }^{\circ} \mathrm{C}$ ) and velocity fields around (a) horizontal-elliptical conductor, and (b) vertical-elliptical conductor (Al strands of $1.83 \mathrm{~mm}$, current of $800 \mathrm{~A}$ and wind velocity of $1 \mathrm{~m} / \mathrm{s}$ ). The Sun is at the zenith.

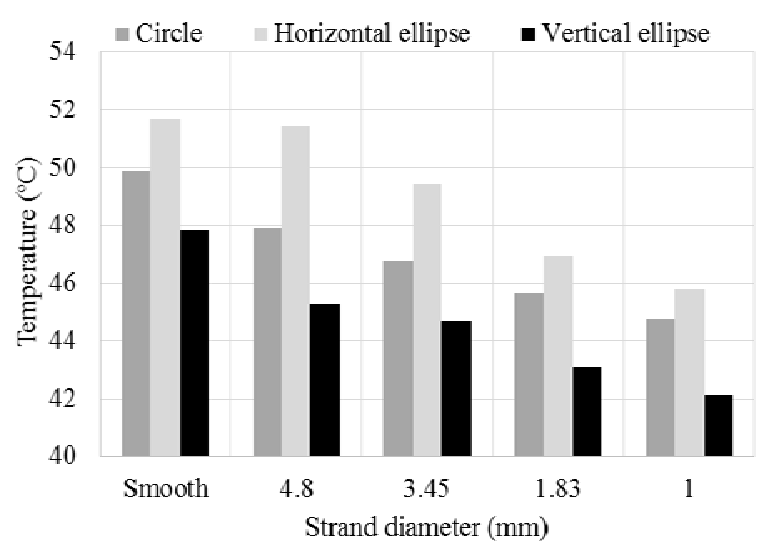

Fig. 8. Maximum temperature for different strand diameter in circular and elliptical conductors (current of $800 \mathrm{~A}$ and wind velocity of $1 \mathrm{~m} / \mathrm{s}$ )

For the present study case, Fig. 8 shows the maximum temperature achieved with each conductor shape as a function of the strand diameter, where "smooth" denotes circular or elliptical shapes with solid section. As can be observed, the vertical-elliptical conductor can provide lower temperature than the circular conductor for the smooth case, while the temperature in the horizontal one is much higher. This is also observed for stranded-conductor surfaces. The main reason of this behavior is that, for the moderate load level considered in the line (800 A), heat evacuation by radiation and forced convection are more or less in the same order for all conductor shapes. However, the surface exposed to solar radiation is higher in the horizontal-elliptical conductor (the Sun is supposed to be at the zenith). Hence, the heat input on this conductor is noticeably higher than in the other conductor shapes, leading to a higher temperature.

Also, as concluded previously, the use of thinner strands leads to lower temperatures, even lower than the smooth case. This is also shown in Fig. 9 for the particular case of the vertical-elliptical conductor, where it is depicted the evolution of the maximum temperature of the conductor as a function of strand diameter and wind velocity. It is easily observed that, for a given wind velocity, the temperature of the conductor may be about $2.8{ }^{\circ} \mathrm{C}$ higher when using strands of $4.8 \mathrm{~mm}$ instead of $1.83 \mathrm{~mm}$. However this effect is only of importance for wind velocities in the range of $0.5-3 \mathrm{~m} / \mathrm{s}$.

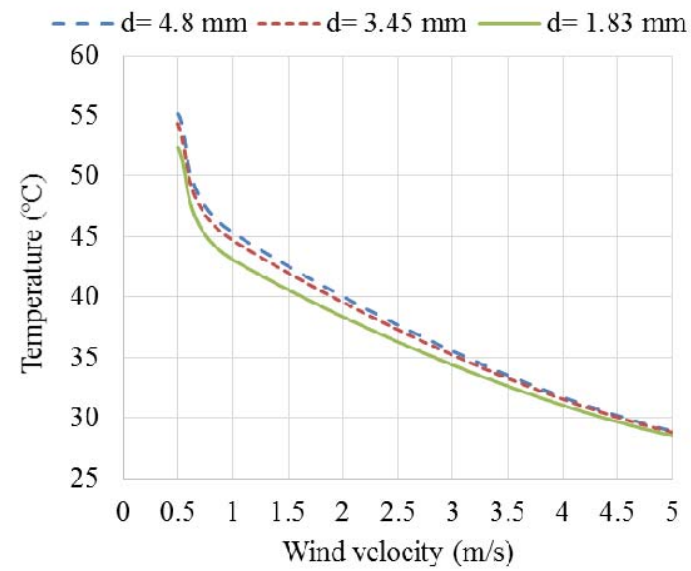

Fig. 9. Influence of $\mathrm{Al}$ strands diameter on the maximum temperature of the vertical-elliptical conductor for different wind velocities (current of $800 \mathrm{~A}$ ).

On the other hand, it is of importance to analyze the influence of strand diameter, hence the surface design, on the heat evacuation for different load levels in the conductor. This is shown in Fig. 10 for the case of a vertical-elliptical conductor, where it is easily concluded that thinner strands leads to a lower temperature specially for important load levels. In particular, for a wind velocity of $1 \mathrm{~m} / \mathrm{s}$ (Fig. 10a), the difference between using strands of $4.8 \mathrm{~mm}$ and $1.83 \mathrm{~mm}$ is in the range of $8{ }^{\circ} \mathrm{C}$ for a current of 1600 A. However, this behaviour is less important when wind speed increases. In this sense, for a wind velocity of $5 \mathrm{~m} / \mathrm{s}$ (Fig. 10b), higher currents can flow through the conductors, but the differences decrease up to $5{ }^{\circ} \mathrm{C}$ for the range of strands diameter considered. Nonetheless, it has to be noticed that this effect starts to be of importance (differences higher than $3{ }^{\circ} \mathrm{C}$ ) for currents higher than $1000 \mathrm{~A}$ for wind velocities of about $1 \mathrm{~m} / \mathrm{s}$, and $2000 \mathrm{~A}$ in the case of wind velocities in the range of $5 \mathrm{~m} / \mathrm{s}$.

Although previous results conclude that a verticalelliptical conductor would be the best choice in order to reduce the maximum temperature of an overhead 
conductor, it is of interest to analyse if this conclusion is always true in relation with the other conductor shapes. In this sense, Fig. 11 represents the influence of the load level for different strand diameters in the cases of circular (Cx.x), horizontal-elliptical (Hx.x) and vertical-elliptical (Vx.x) conductors (where x.x denotes strand diameters of $4.8 \mathrm{~mm}, 3.45 \mathrm{~mm}$ and $1.83 \mathrm{~mm}$ ). As can be observed, for strand diameters of $4.8 \mathrm{~mm}$ and $3.45 \mathrm{~mm}$, the verticalelliptical conductor is always the coolest conductor shape, although the differences on the temperature are almost negligible when the conductor is close to its thermal limit $\left(100^{\circ} \mathrm{C}\right)$. However, for a strand diameter of $1.83 \mathrm{~mm}$ this is not always true, since it is easily observed how the lowest temperature is achieved by the horizontal-elliptical conductor when the current is higher than $1650 \mathrm{~A}$. The main reason of this behavior is again related to the importance of each heat transfer mechanism for the load levels considered. In this particular situation, when conductors are close to their thermal limit (around $100{ }^{\circ} \mathrm{C}$ ), the heat evacuated by radiation is more important than the heat input caused by solar radiation. As a consequence, heat transfer by forced convection makes now the difference, and this mechanism is more efficient in horizontal-elliptical conductors made by thin strands, which increase the external surface of the conductor exposed to forced convection.

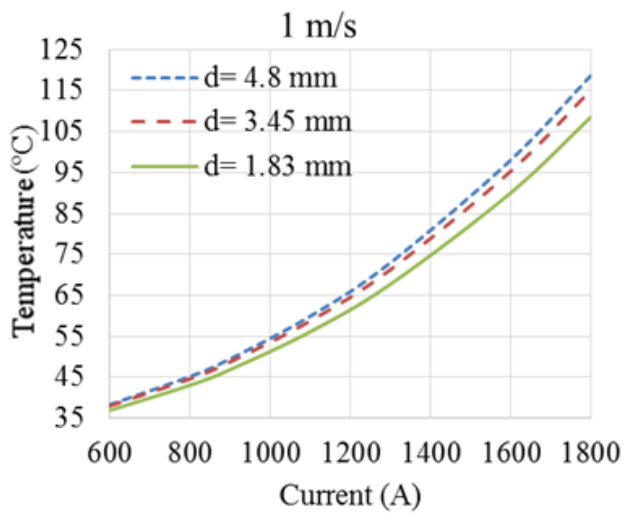

(a)

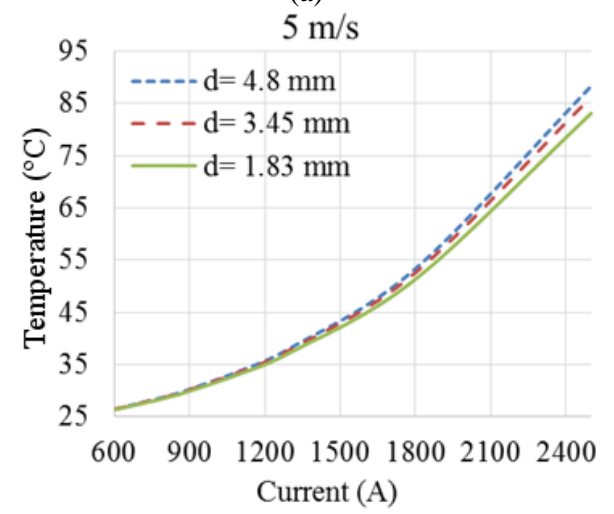

(b)

Fig. 10. Evolution of the temperature in the vertical-elliptical conductor as a function of the strand diameter and conductor current for a wind velocity of $1 \mathrm{~m} / \mathrm{s}$ (a) and $5 \mathrm{~m} / \mathrm{s}$ (b).

These results show that a deeper analysis is required, since the optimal conductor shape may depend on environmental and technical conditions. However, in a first approach it can be concluded that the vertical-elliptical conductor would be the first choice to be considered. For this reason, it is also of interest to analyze the influence of the air temperature on the temperature achieved by this conductor shape. This is shown in Fig. 12 for a wind velocity of $1 \mathrm{~m} / \mathrm{s}$ and a current of $800 \mathrm{~A}$. As can be observed, the air temperature has some influence on the variations of the heat evacuation derived from the use of thicker or thinner strands. In this sense, for the particular situation shown in Fig. 12, there is a difference of about 2 ${ }^{\circ} \mathrm{C}$ when using strands of $4.8 \mathrm{~mm}$ instead of $1.83 \mathrm{~mm}$ for air temperatures ranging from $20^{\circ} \mathrm{C}$ to $40{ }^{\circ} \mathrm{C}$. However, this is only noticeable for wind velocities in the order of $1 \mathrm{~m} / \mathrm{s}$, since the differences observed for higher values of wind velocities are lower than $0.3^{\circ} \mathrm{C}$.

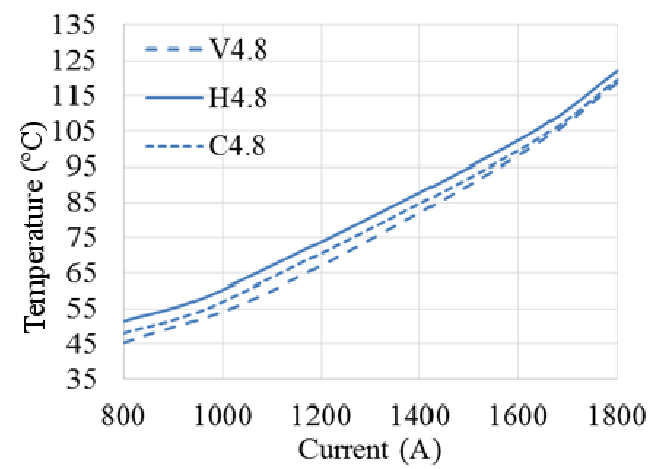

(a)

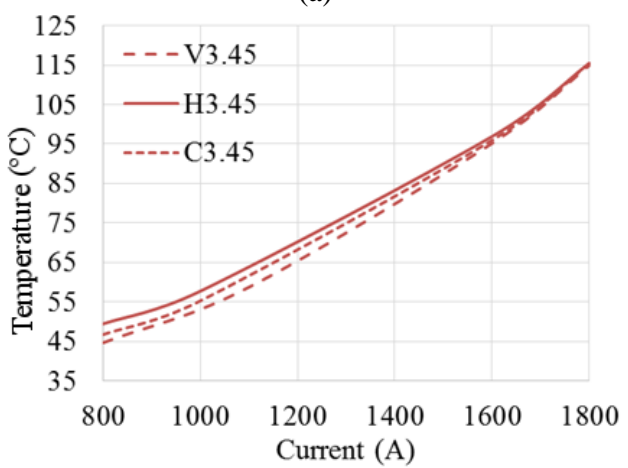

(b)

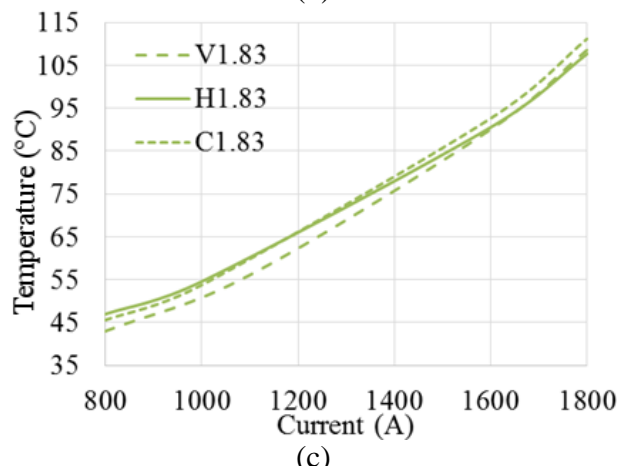

Fig. 11. Influence of load level for different conductor shapes and strand diameter: (a) $4.8 \mathrm{~mm}$, (b) $3.45 \mathrm{~mm}$ and (c) $1.83 \mathrm{~mm}$. 


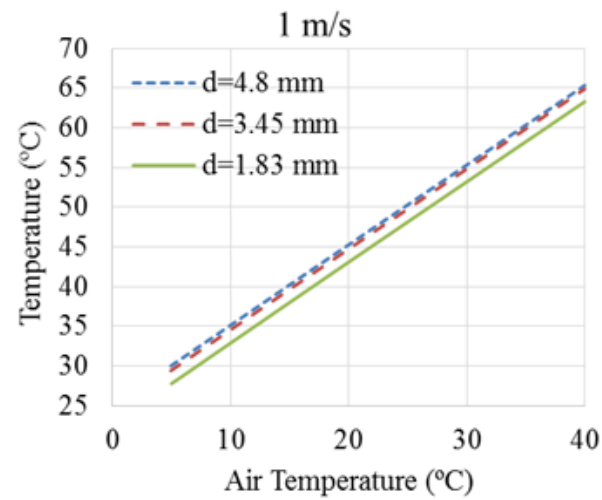

Fig. 12. Influence of the air temperature and the strand diameter on the temperature of the vertical-elliptical conductor for a wind velocity of $1 \mathrm{~m} / \mathrm{s}$ (current of $800 \mathrm{~A}$ ).

\section{Conclusions}

Novel designs have been proposed to improve the heat convection transfer of overhead conductors with the aim of increasing the thermal limit. The effect of the strand diameter and the conductor shape on the thermal behaviour have been analyzed, concluding that the smaller strand diameter the lower the steady-state temperature. This effect is more pronounced at low wind speeds of around 1 $\mathrm{m} / \mathrm{s}$. This behaviour is also valid for the elliptical stranded conductor, together with an additional advantage derived from the shape, being the vertical arrangement more advantageous than the horizontal one. A reduction of around $5{ }^{\circ} \mathrm{C}$ can be obtained for a section similar to ACSR Condor when the customary rounded shape stranded conductor is replaced by the vertical-elliptical one, resulting in increase of around $7 \%$ of thermal rating. Further research regarding the 3-D design of the conductor and its thermal and mechanical behaviour will be carried out in the next future with the expectancy of finding a design that have a significantly better thermal and mechanical behaviour at a reasonable cost.

\section{Acknowledgement}

This work has been supported by the Spanish Ministry of Science \& Innovation under grant ENE2013-48428C2-1-R.

\section{References}

[1] A. Useros and S. Fernández, "More Capacity Allows More Renewables", Transmission and Distribution World, June 2015.

[2] D. Johnson, "High-performance Conductor Means Much Greater Capacity”, Electricity Today, September 2006.

[3] WG. B2.12, "Guide for Selection of Weather Parameters for Bare Overhead Conductor Ratings", Cigré TB 299, 2006.

[4] A. Bergstrom, U. Axelsson and V. Neimane, "Dynamic capacity rating for wind cooled overhead lines", in Proc. CIRED, pp. 1-5, Stockholm, June 2013.

[5] J. C. del-Pino-López, D. Garrido-García, P. Cruz-Romero and A. Gómez-Expósito, "Influence of Overhead Power Conductor Shape on its Temperature”, in Proc. $2^{\text {nd }}$ Iberian Comsol Conference, Málaga, June 2015.

[6] COMSOL Multiphysics Reference Manual, Version: October 2014 COMSOL 5.1.

[7] T. Saito, Y. Hase, et. al., "Spiral-elliptic conductor with low-drag coefficient", in Proc. Power Engineering Society Winter Meeting, Vol. 4, pp. 2397-2402, January 2000.

[8] L. Konti, "A proposed algorithm for an overhead transmission line conductor temperature rise calculation", International Transactions on Electrical Energy Systems, Volume 24, pp. 578-596, 2014.

[9] L. Chen, Z. Zheng, et. al., “Thermal grading around overhead transmission line under various environments and its influence to load capacity", in Proc. International Symposium Electrical Insulation, pp. 545-548, San Juan, June 2012.

[10] IEC 62219:2002, “Overhead electrical conductors. Formed wire, concentric lay, stranded conductors”. 